УДК 661.152 .4

\title{
ОСОБЕННОСТИ ПРОЦЕССА ГРАНУЛИРОВАНИЯ ОРГАНОМИНЕРАЛЬНЫХ УДОБРЕНИЙ МЕТОДОМ ОКАТЫВАНИЯ
}

\author{
Сковородников Павел Валерьевич', \\ pavel.skovorodnikov@yandex.ru \\ Черепанова Мария Владимировна', \\ syromyatnikova.maria@yandex.ru \\ 1 Пермский национальный исследовательский политехнический университет,
Россия, 614990, г. Пермь, Комсомольский пр., 29.
}

Актуальность. Использование органоминеральных удобрений позволяет существенно повысить плодородие почвы и восстановить ее агрохимические и физические свойства. Исследование позволит решить некоторые проблемы, связанные с применением удобрения в необработанном виде, а полученный продукт в виде сферических гранул размером от 2,0 до 5, 5 мм будет иметь высокую статическую прочность и может быть легко использован в сельском хозяйстве в условиях сплошного внесения удобрений в почву с использованием агротехники.

Цель: изучение процесса гранулирования органоминерального удобрения методом окатывания с предварительной подформовкой и определение оптимальных технологических параметров; исследование вероятности образования труднорастворимых соединений при взаимодействии растворов связующих с компонентами, входящими в состав органоминеральных удобрений. Объект: органоминеральное удобрение биогумус с влажностью 55-60\%.

Методы: электронная сканирующая микроскопия (Hitachi «S-3400N»), позволяющая определить элементный состав биогумуса и оценить поверхность частиц удобрения и готового гранулированного продукта; рентгеноспектральный анализ, используемый для установления элементного состава; термодинамический анализ, необходимый для оценки вероятности протекания реакций и возможности образования труднорастворимых соединений в гранулированном удобрении, способствующих увеличению прочности гранул; ситовой анализ, используемый для определения гранулометрического состава, и метод определения статической прочности гранул на приборе ИПГ-1М.

Результаты. С помощью электронно-сканирующего микроскопа была изучена и описана поверхность частиц органоминерального удобрения - биогумус. Установлен элементный состав органоминерального удобрения: C, N, P, K, O, Fe, Al, Ca, Mg, Na, Cl, S, Ti. Определены оптимальные параметры технологии гранулирования органоминерального удобрения с предварительным формованием через ячейки диаметром 4,0 мм: продолжительность 180 с, температура окатывания $25^{\circ} \mathrm{C}$, сушка при $110^{\circ} \mathrm{C}$ в течение 60 мин и величина ретура 3 \%. Определен вид связующего раствора и его содержание в тукосмеси, благодаря которому можно получить продукт с наилучшими товарными характеристиками (статическая прочность, выход товарной фракции от 2,0 до 5,5 мм). На основании термодинамического анализа установлена возможность протекания химических реакций между связующим раствором и компонентами биогумуса, в результате которых образуются труднорастворимые соединения $\left(\mathrm{Mg}_{3}\left(\mathrm{PO}_{4}\right)_{2}, \mathrm{Ca}_{3}\left(\mathrm{PO}_{4}\right)_{2}, \mathrm{Fe}_{3}\left(\mathrm{PO}_{4}\right)_{2}\right)$, способствующие увеличению прочности гранул. Применяемый в качестве связующего вещества водный раствор фосфата натрия позволяет получить гранулы, обладающие высокой статической прочностью (51,1 Н/гранула). С помощью электронно-сканирующей микроскопии изучена и описана структура получаемого гранулированного удобрения.

\section{Ключевые слова:}

Гранулирование, органоминеральное удобрение, биогумус, окатывание,

связующий раствор, метасиликат натрия, фосфат натрия.

\section{Введение}

На фоне непростых отношений России с другими странами и введённого режима санкций в различных областях производства остро стоит вопрос импортозамещения. Это касается и продуктов сельскохозяйственного назначения. Необходимо производить достаточное количество продуктов растительного происхождения для удовлетворения потребностей населения за счет увеличения урожайности. Данный эффект может быть достигнут при использовании удобрений, позволяющих не только восстановить полезную микрофлору, но и повысить плодородие почвы.

Одним из высокоэффективных средств, улучшающих агрохимические свойства почвы, является органоминеральное удобрение (ОМУ), такое как биогумус, произведенное по инновационной технологии вермикомпостирования, которая основана на биологической переработке органических отхо- дов с применением определенного вида червей. При этом чаще всего используются красные калифорнийские черви (красные вигглеры), которые редко встречаются в почве и легко адаптируются к условиям в гниющей растительности и среде [1-3].

В качестве сырья для производства биогумуса могут использоваться: перегной, навоз, торф, сапропель, бытовые и производственные отходы. В научно-технической и патентной литературе приведено большое количество видов сырья, которое можно использовать для изготовления биогумуса: куриный помет, известь, торф $[4,5]$; золу $[6,7]$; сапропель, торф, минеральные удобрения [8-12] и т. д.

Однако применение биогумуса в необработанном виде, представляющем собой почвообразную массу, вызывает некоторые проблемы. При нарушении условий хранения готового ОМУ происходит ухудшение качества продукта. Из-за высокого 
влагосодержания биогумус слеживается, что приводит к трудностям при транспортировке, а также затрудняет дозирование при внесении в почву. В связи с этим целью работы являлось исследование процесса гранулирования биогумуса методом окатывания с применением предварительной стадии формования.

\section{Материалы и метод исследования}

Объектом исследований являлся биогумус, имеющий нейтрально-щелочную реакцию среды $(\mathrm{pH}=7,2-8,1)$, с общим содержанием влаги $55-60 \%$. Основным компонентом ОМУ являются гуминовые кислоты, также в его состав входят: азот, фосфор, калий, магний, кальций. Химический состав биогумуса приведен в табл. 1.

Таблица 1. Хилический состав ОМУ биогумус

Table 1. Chemical composition of OMF biohumus

\begin{tabular}{|c|c|c|c|c|c|c|}
\hline $\begin{array}{l}\text { Показатель } \\
\text { Index }\end{array}$ & $\begin{array}{l}\text { Азот } \\
(\mathrm{N})\end{array}$ & $\begin{array}{c}\text { Фосфор } \\
\left(\mathrm{P}_{2} \mathrm{O}_{5}\right)\end{array}$ & $\begin{array}{c}\text { Калий } \\
\left(\mathrm{K}_{2} \mathrm{O}\right)\end{array}$ & $\begin{array}{c}\text { Магний } \\
(\mathrm{Mg})\end{array}$ & $\begin{array}{c}\text { Каль- } \\
\text { ций } \\
(\mathrm{Ca})\end{array}$ & 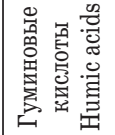 \\
\hline $\begin{array}{c}\text { Граничные } \\
\text { нормы, \% мac. } \\
\text { Boundary } \\
\text { norms, wt. \% }\end{array}$ & $0,8-2,0$ & $0,8-2,0$ & $0,7-1,2$ & $0,3-0,5$ & $2,0-3,0$ & $25,0-30,0$ \\
\hline
\end{tabular}

Структура поверхности частиц биогумуса, полученная с использованием электронного сканирующего микроскопа Hitachi «S-3400N» (Япония), оснащенного энергодисперсионным спектрометром «XFlash 4010» фирмы «Bruker» (Германия), изображена на рис. 1.

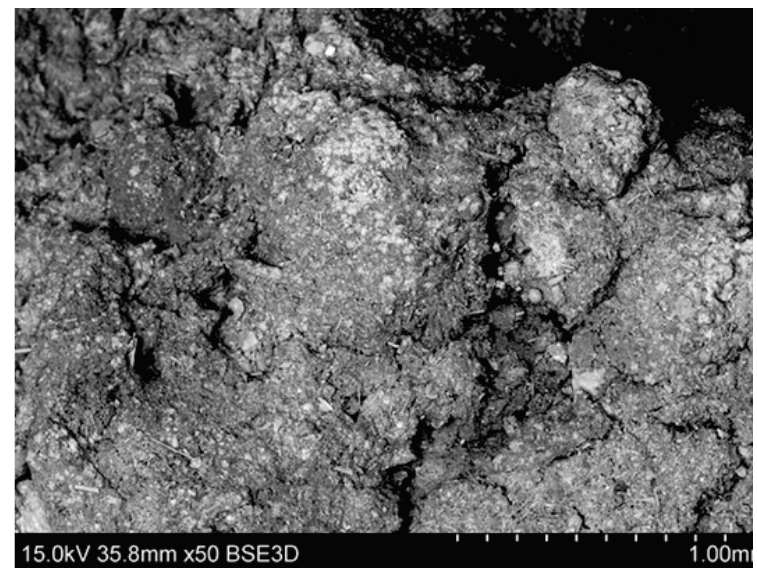

Pис.1. Микрофотография поверхности частии биогумуса (увеличение х50)

Fig. 1. Microphotography of biohumus particle surface (magnification $\times 50$ )

Видно, что структура поверхности частицы биогумуса неоднородная, состоящая из различных игольчатых и шарообразных вкраплений, с рыхлой упаковкой. Некоторые участки частицы покрыты налетом светлого цвета.

C помощью рентгеноспектрального анализа установили элементный состав, результаты которого представлены на рис. 2.

Биогумус состоит из разнообразных элементов: K, $\mathrm{Ca}, \mathrm{P}, \mathrm{C} 1, \mathrm{Mg}, \mathrm{Fe}$ и т. д. Основными элементами биогумуса являются: углерод 18-30\%, кислород 40-50\% и кремний 8-11\%.

Исходя из результатов проведенного анализа, можно предположить наличие следующих ве-

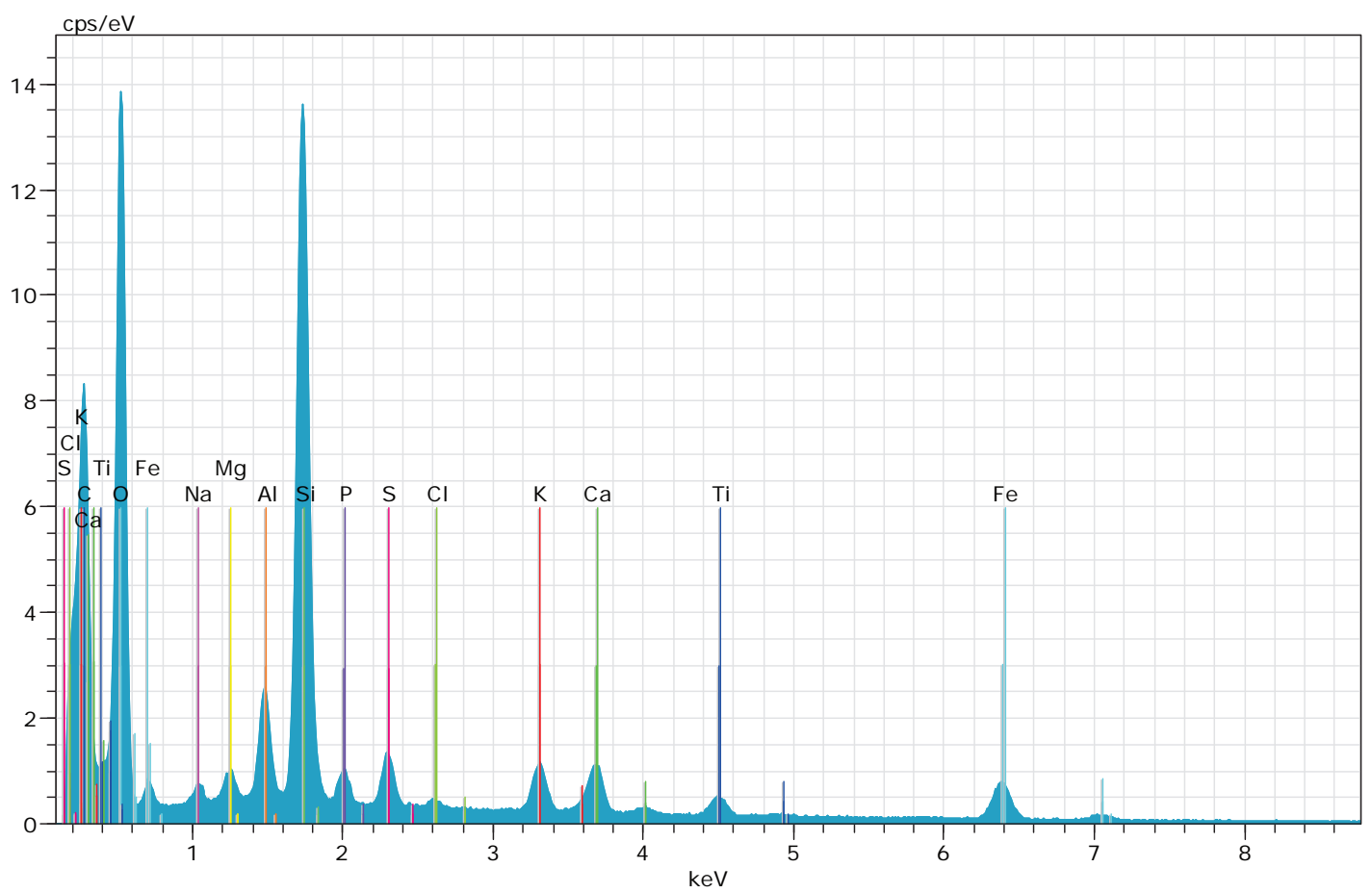

Pис. 2. Результаты элементного анализа биогумуса

Fig. 2. Results of elemental analysis of biohumus 
ществ в составе ОМУ: $\mathrm{SiO}_{2}, \mathrm{Al}_{2} \mathrm{O}_{3}, \mathrm{Na}_{2} \mathrm{SO}_{4}, \mathrm{MgSO}_{4}$, $\mathrm{NaCl}, \mathrm{CaCO}_{3}, \mathrm{CaHPO}_{4}, \mathrm{Ca}\left(\mathrm{H}_{2} \mathrm{PO}_{4}\right)_{2}, \mathrm{MgCl}_{2}, \mathrm{CaCl}_{2}$, $\mathrm{TiO}_{2}, \mathrm{Fe}_{2} \mathrm{O}_{3}, \mathrm{KAlSi}_{3} \mathrm{O}_{8}, \mathrm{FeSO}_{4}$ и др.

Существует большое количество способов и методов гранулирования различных материалов. Однако нет единого способа, предназначенного для гранулирования биогумуса, хотя могут применяться такие методы, как:

- формование или экструзия;

- таблетирование;

- прессование;

- окатывание [13].

Исследования по гранулированию ОМУ проводили на установке, включающей: экструдер с заданным размером ячеек матрицы, барабанный гранулятор со скоростью вращения до 40 об/мин, имеющий электрический привод и сушильный шкаф.

Благодаря проведенным опытным испытаниям была предложена методика проведения эксперимента по изучению процесса гранулирования биогумуса, а также определены основные технологические параметры процесса. По разработанной методике к исходной порошкообразной смеси ОМУ добавляли водный раствор связующего вещества, полученную смесь перемешивали и при помощи экструдера формовали через матрицу с ячейками 4,0 мм. Далее материал направляли в барабанный гранулятор, который оснащен рубашкой для поддержания требуемых температур [14]. Гранулирование методом окатывания проводили в течение экспериментально установленного времени и при ранее установленной температуре. Затем готовое гранулированное удобрение направляли в сушильный шкаф, где при температуре $110{ }^{\circ} \mathrm{C}$ проводили сушку нагретым потоком воздуха. После окончания сушки определяли гранулометрический состав готового продукта. Качество продукта и эффективность гранулирования при исследуемых параметрах определяли по нескольким показателям: статическая прочность, выход товарной фракции (более 2,0 мм и менее 5,5 мм) $[15,16]$.

\section{Результаты исследования}

Применение метода окатывания при гранулировании ОМУ позволяет получить однородные гранулы, обладающие правильной шарообразной формой, уменьшить слеживаемость и предотвратить разрушение продукта при транспортировании и внесении в почву. Следовательно, важным параметром проведения гранулирования является продолжительность окатывания. Данное исследование проводили при комнатной температуре и временном диапазоне от 60 до 360 с, гранулированное удобрение сушили при $110{ }^{\circ} \mathrm{C}$. Полученные результаты представлены в табл. 2.

На основании полученных данных видно, что продолжительность окатывания оказывает влияние на характеристики гранулированного продукта. При продолжительном гранулировании увеличивался выход гранул товарной фракции
$(-5,5+2,0$ мм). Так, при значении 180 секунд наблюдается максимальный выход готового гранулированного удобрения $-87,97 \%$, значение статической прочности гранул составляет 19,4 Н/гранула. Дальнейшее увеличение продолжительности окатывания введет к снижению выхода продукта. Это происходит за счет активного слипания частиц ОМУ между собой, что способствует одновременному образованию крупных гранул нетоварной фракции более 5,5 мм, а также разрушению товарной фракции с образованием гранул менее 2,0 мм. Таким образом, оптимальным временем окатывания является $180 \mathrm{c.}$

Таблица 2. Характеристика гранул биогумуса в зависимости от продолжительности гранулирования окатыванием

Table 2. Characteristics of biohumus granules depending on $d u$ ration of granulation by pelletizing

\begin{tabular}{|c|c|c|c|c|c|c|c|}
\hline \multirow{3}{*}{ 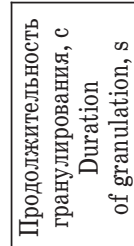 } & \multicolumn{4}{|c|}{$\begin{array}{c}\text { Гранулометрический } \\
\text { состав, \% } \\
\text { Granulometric } \\
\text { composition, } \%\end{array}$} & \multirow{3}{*}{ 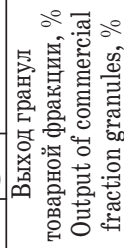 } & \multirow{3}{*}{ 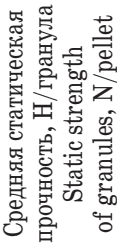 } & \multirow{3}{*}{ 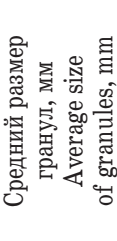 } \\
\hline & $+5,5$ & \begin{tabular}{|l|}
$-5,5$ \\
$+2,0$ \\
\end{tabular} & $\begin{array}{r}-2,0 \\
+1,0 \\
\end{array}$ & \begin{tabular}{|l}
$-1,0$ \\
$+0,0$ \\
\end{tabular} & & & \\
\hline & \multicolumn{4}{|c|}{$\mathrm{MM} / \mathrm{mm}$} & & & \\
\hline 60 & 5,88 & 87,07 & 3,71 & 3,35 & 87,07 & $18,0 \pm 0,3$ & 3,73 \\
\hline 120 & 5,73 & 87,49 & 3,42 & 3,37 & 87,49 & $18,5 \pm 0,3$ & 3,64 \\
\hline 180 & 5,65 & 87,97 & 3,28 & 3,10 & 87,97 & $19,4 \pm 0,2$ & 3,70 \\
\hline 240 & 5,28 & 87,92 & 4,03 & 2,78 & 87,92 & $19,2 \pm 0,3$ & 3,69 \\
\hline 300 & 6,17 & 87,20 & 3,37 & 3,26 & 87,20 & $19,7 \pm 0,6$ & 3,71 \\
\hline 360 & 6,01 & 86,97 & 3,55 & 3,46 & 86,97 & $19,0 \pm 0,3$ & 3,68 \\
\hline
\end{tabular}

При повышенных температурах должно происходить увеличение прочности гранул и уменьшение влажности в продукте, поступающем на сушку. Это объясняется увеличением эффективности гранулообразования вследствие выравнивания поля температур внутри гранул и уменьшения переноса влаги внутрь гранулы за счет перепада температур $[17,18]$. Следствием этого является увеличение производительности технологической установки.

Для подтверждения приведенных выше теоретических предпосылок была проведена серия исследований, направленных на установление влияния температуры на процесс формирования гранул. Температурный интервал от 25 до $90^{\circ} \mathrm{C}$.

Исследования проводили с использованием предварительного формования. При продолжительности окатывания в барабанном грануляторе 180 секунд и температуре сушки $110{ }^{\circ} \mathrm{C}$. Результаты исследования представлены на рис. 3.

Было выявлено, что в результате увеличения температуры процесса гранулирования методом окатывания происходит изменение товарных характеристик продукта. Хотя данный параметр и оказывает положительное влияние, увеличение показателей качества гранулированного биогумуса незначительно. Так, при температуре $90{ }^{\circ} \mathrm{C}$ максимальное значение выхода гранул товарной фракции составляет $88,51 \%$ и статическая прочность 
20,55 Н/гранула. В общем статическая прочность при высоких температурах окатывания увеличилась на $5,5 \%(1,15 \mathrm{H} /$ гранула) и выход на $0,5 \%$. На основании имеющихся данных о влиянии температуры процесса гранулирования методом окатывания было установлено, что целесообразнее применять более низкую температуру. Это позволит снизить текущие энергозатраты и сделать технологию менее трудоемкой, а повышения прочности можно добиться за счет введения связующих веществ или упрочняющих добавок, которые будут способствовать дополнительному обогащению питательными веществами. Таким образом, при температуре окатывания $25{ }^{\circ} \mathrm{C}$ значения статической прочности и выхода товарной фракции составили 19,4 Н/гранула и 87,97 \%, соответственно.

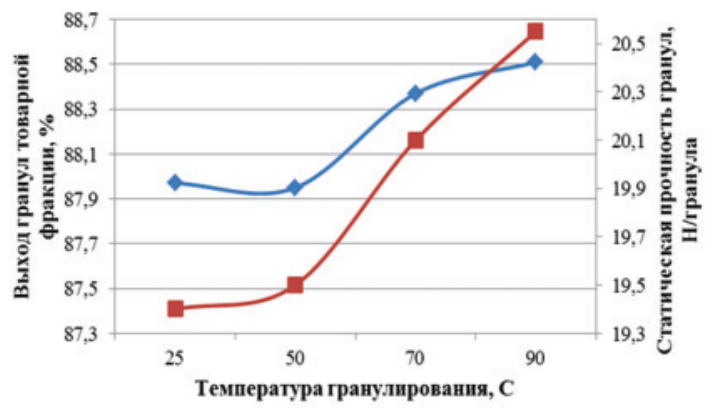

$\rightarrow$ Выход гранул товарной фракциш, \% $\rightarrow$-Статическая прочность гранул, Н/гранула

Pис. 3. Влияние телпературы окатывания на характеристики гранул биогумуса

Pig. 3. Influence of rolling temperature on characteristics of biohumus granules

В процессе гранулирования возможно применение различных растворов веществ в качестве связующих. Это может оказать существенное влияние на физико-механические характеристики готового продукта. При добавлении жидкости внутрь исследуемого образца капля под действием капиллярных сил распространяется в объеме смеси, заполняя поры между частицами, когда влажность достигает максимальной капиллярной влагоемкости, распространение в сыпучей смеси прекращается $[19,20]$. Проводилось изучение влияния вида и содержания в тукосмеси водных растворов связующих, в качестве которых использовали 5-водный метасиликат и 12-водный фосфат натрия с концентрациями $10,20,30 \%$. Результаты исследования представлены в табл. 3.

Применение водных растворов связующих веществ оказывает положительное влияние на характеристики гранулированного продукта. При содержании 5 \% в тукосмеси 30 \% водного раствора метасиликата натрия достигается максимальное значение статической прочности $24,1 \mathrm{H} /$ гранула и максимальный выход гранул товарной фракции $96,17 \%$.

Использование водного раствора связующего фосфата натрия способствует увеличению товарных характеристик готового продукта, при содер- жании $5 \%$ в тукосмеси $30 \%$ раствора максимальное значение составляет 51,1 Н/гранула и выход товарной фракции 94,46\% .

Таблица 3. Влияние вида и содержания водных растворов связующих на характеристики гранулированного биогулуса

Table 3. Influence of the type and content of aqueous solutions of binders on characteristics of granulated biohumus

\begin{tabular}{|c|c|c|c|c|c|}
\hline \multirow{3}{*}{ 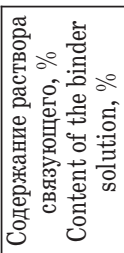 } & \multicolumn{2}{|c|}{$\begin{array}{c}\text { Гранулометрический } \\
\text { состав, \% } \\
\text { Granulometric } \\
\text { composition, } \% \\
\end{array}$} & \multirow{3}{*}{ 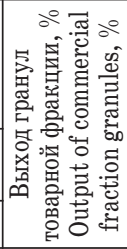 } & \multirow{3}{*}{ 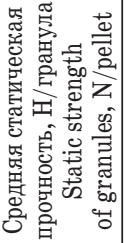 } & \multirow{3}{*}{ 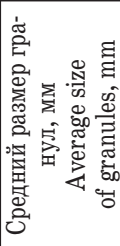 } \\
\hline & $+5,5 \mid \begin{array}{l}-5,5 \\
+2,0\end{array}$ & \begin{tabular}{|l|l|}
$-2,0$ & $-1,0$ \\
$+1,0$ & $+0,0$ \\
\end{tabular} & & & \\
\hline & \multicolumn{2}{|c|}{$\mathrm{MM} / \mathrm{mm}$} & & & \\
\hline \multicolumn{6}{|c|}{ Без связующего/Without binder } \\
\hline- & \begin{tabular}{l|l|}
5,65 & 87,97 \\
\end{tabular} & \begin{tabular}{|l|l|}
3,28 & 3,10 \\
\end{tabular} & 87,97 & $19,4 \pm 0,2$ & 3,70 \\
\hline \multicolumn{6}{|c|}{$\begin{array}{c}10 \% \text { водный pacmвор } \mathrm{Na}_{2} \mathrm{SiO}_{3} \cdot 5 \mathrm{H}_{2} \mathrm{O} \\
10 \% \text { water solution } \mathrm{Na}_{2} \mathrm{SiO}_{3} \cdot 5 \mathrm{H}_{2} \mathrm{O}\end{array}$} \\
\hline 1 & \begin{tabular}{l|l|}
1,87 & 91,12 \\
\end{tabular} & \begin{tabular}{|l|l|}
3,45 & 3,56 \\
\end{tabular} & 91,12 & $18,6 \pm 0,2$ & 3,50 \\
\hline 2 & \begin{tabular}{l|l|}
2,80 & 91,94 \\
\end{tabular} & \begin{tabular}{|l|l|}
3,00 & 2,26 \\
\end{tabular} & 91,94 & $18,9 \pm 0,3$ & 3,57 \\
\hline 3 & \begin{tabular}{l|l|}
2,32 & 92,46 \\
\end{tabular} & \begin{tabular}{|l|l|}
2,56 & 2,66 \\
\end{tabular} & 92,46 & $19,2 \pm 0,5$ & 3,55 \\
\hline 4 & \begin{tabular}{l|l|}
2,56 & 93,15
\end{tabular} & \begin{tabular}{|l|l|}
1,98 & 2,31
\end{tabular} & 93,15 & $19,9 \pm 0,4$ & 3,58 \\
\hline 5 & \begin{tabular}{l|l|}
2,84 & 93,87 \\
\end{tabular} & \begin{tabular}{|l|l|}
1,88 & 1,41 \\
\end{tabular} & 93,87 & $20,2 \pm 0,2$ & 3,62 \\
\hline \multicolumn{6}{|c|}{$\begin{array}{c}20 \% \text { водный pacmвор } \mathrm{Na}_{2} \mathrm{SiO}_{3} \cdot 5 \mathrm{H}_{2} \mathrm{O} 20 \\
\% \text { water solution } \mathrm{Na}_{2} \mathrm{SiO}_{3} \cdot 5 \mathrm{H}_{2} \mathrm{O}\end{array}$} \\
\hline 1 & \begin{tabular}{l|l|}
4,17 & 91,98 \\
\end{tabular} & \begin{tabular}{|l|l|}
2,96 & 0,89 \\
\end{tabular} & 91,98 & $20,5 \pm 0,3$ & 3,59 \\
\hline 2 & \begin{tabular}{l|l|}
3,76 & 93,45 \\
\end{tabular} & \begin{tabular}{|l|l|}
2,56 & 0,23 \\
\end{tabular} & 93,45 & $20,8 \pm 0,4$ & 3,66 \\
\hline 3 & \begin{tabular}{l|l|}
2,96 & 93,99 \\
\end{tabular} & \begin{tabular}{|l|l|}
2,78 & 0,59 \\
\end{tabular} & 93,99 & $20,9 \pm 0,4$ & 3,64 \\
\hline 4 & \begin{tabular}{l|l|}
2,76 & 94,62 \\
\end{tabular} & \begin{tabular}{|l|l|}
1,67 & 0,95 \\
\end{tabular} & 94,62 & $21,8 \pm 0,3$ & 3,64 \\
\hline 5 & \begin{tabular}{|l|l|}
3,24 & 94,12 \\
\end{tabular} & \begin{tabular}{|l|l|}
1,71 & 0,93 \\
\end{tabular} & 94,12 & $20,3 \pm 0,4$ & 3,64 \\
\hline \multicolumn{6}{|c|}{$\begin{array}{l}30 \% \text { водный pacmвор } \mathrm{Na}_{2} \mathrm{SiO}_{3} \cdot 5 \mathrm{H}_{2} \mathrm{O} \\
30 \% \text { water solution } \mathrm{Na}_{2} \mathrm{SiO}_{3} \cdot 5 \mathrm{H}_{2} \mathrm{O}\end{array}$} \\
\hline 1 & \begin{tabular}{l|l|}
2,98 & 92,23 \\
\end{tabular} & \begin{tabular}{|l|l|}
2,54 & 2,25 \\
\end{tabular} & 92,23 & $21,1 \pm 0,4$ & 3,58 \\
\hline 2 & \begin{tabular}{l|l|}
2,26 & 93,96 \\
\end{tabular} & \begin{tabular}{|l|l|}
2,94 & 0,84 \\
\end{tabular} & 93,96 & $21,5 \pm 0,3$ & 3,56 \\
\hline 3 & \begin{tabular}{l|l|}
2,49 & 94,31 \\
\end{tabular} & \begin{tabular}{|l|l|}
2,68 & 0,52 \\
\end{tabular} & 94,31 & $21,9 \pm 0,3$ & 3,58 \\
\hline 4 & \begin{tabular}{|l|l|}
2,78 & 96,08 \\
\end{tabular} & \begin{tabular}{|l|l|}
0,78 & 0,36 \\
\end{tabular} & 96,08 & $22,8 \pm 0,3$ & 3,61 \\
\hline 5 & \begin{tabular}{|l|l|}
3,03 & 96,17 \\
\end{tabular} & \begin{tabular}{|l|l|}
0,57 & 0,23 \\
\end{tabular} & 96,17 & $24,1 \pm 0,2$ & 3,60 \\
\hline \multicolumn{6}{|c|}{$\begin{array}{c}10 \% \text { водный pacmвор } \mathrm{Na}_{3} \mathrm{PO}_{4} \cdot 12 \mathrm{H}_{2} \mathrm{O} \\
10 \% \text { water solution } \mathrm{Na}_{3} \mathrm{PO}_{4} \cdot 12 \mathrm{H}_{2} \mathrm{O}\end{array}$} \\
\hline 1 & \begin{tabular}{|l|l|}
1,34 & 93,55 \\
\end{tabular} & \begin{tabular}{|l|l|}
3,03 & 2,08 \\
\end{tabular} & 93,55 & $23,6 \pm 0,2$ & 3,95 \\
\hline 2 & \begin{tabular}{l|l|}
2,80 & 93,72 \\
\end{tabular} & \begin{tabular}{|l|l|}
2,50 & 0,98 \\
\end{tabular} & 93,72 & $24,7 \pm 0,3$ & 3,59 \\
\hline 3 & \begin{tabular}{|l|l|}
2,32 & 93,75 \\
\end{tabular} & \begin{tabular}{|l|l|}
2,64 & 1,29 \\
\end{tabular} & 93,74 & $25,2 \pm 0,5$ & 3,81 \\
\hline 4 & \begin{tabular}{|l|l|}
2,56 & 94,44 \\
\end{tabular} & \begin{tabular}{|l|l|}
2,41 & 0,59 \\
\end{tabular} & 94,44 & $29,9 \pm 0,4$ & 3,51 \\
\hline 5 & \begin{tabular}{|l|l|}
2,84 & 94,73 \\
\end{tabular} & \begin{tabular}{|l|l|}
1,86 & 0,57 \\
\end{tabular} & 94,73 & $32,2 \pm 0,2$ & 4,00 \\
\hline \multicolumn{6}{|c|}{$\begin{array}{c}20 \% \text { водный pacmвор } \mathrm{Na}_{3} \mathrm{PO}_{4} \cdot 12 \mathrm{H}_{2} \mathrm{O} \\
20 \% \text { water solution } \mathrm{Na}_{3} \mathrm{PO}_{4} \cdot 12 \mathrm{H}_{2} \mathrm{O}\end{array}$} \\
\hline 1 & \begin{tabular}{l|l|}
3,84 & 91,13 \\
\end{tabular} & \begin{tabular}{|l|l|}
3,01 & 2,02 \\
\end{tabular} & 91,13 & $25,3 \pm 0,4$ & 4,07 \\
\hline 2 & \begin{tabular}{l|l|}
5,07 & 91,55 \\
\end{tabular} & \begin{tabular}{|l|l|}
2,33 & 1,05 \\
\end{tabular} & 91,55 & $29,8 \pm 0,3$ & 4,23 \\
\hline 3 & \begin{tabular}{l|l|}
2,27 & 94,36 \\
\end{tabular} & \begin{tabular}{|l|l|}
2,32 & 1,05 \\
\end{tabular} & 94,36 & $29,6 \pm 0,3$ & 3,81 \\
\hline 4 & \begin{tabular}{l|l|}
2,88 & 94,55 \\
\end{tabular} & \begin{tabular}{|l|l|}
1,80 & 0,77 \\
\end{tabular} & 94,55 & $29,7 \pm 0,5$ & 3,43 \\
\hline 5 & \begin{tabular}{|l|l|}
2,38 & 94,87 \\
\end{tabular} & \begin{tabular}{|l|l|}
1,87 & 0,88 \\
\end{tabular} & 94,87 & $32,3 \pm, 02$ & 3,73 \\
\hline \multicolumn{6}{|c|}{$\begin{array}{l}30 \% \text { водный pacmвор } \mathrm{Na}_{3} \mathrm{PO}_{4} \cdot 12 \mathrm{H}_{2} \mathrm{O} \\
30 \% \text { water solution } \mathrm{Na}_{3} \mathrm{PO}_{4} \cdot 12 \mathrm{H}_{2} \mathrm{O}\end{array}$} \\
\hline 1 & \begin{tabular}{l|l|}
2,12 & 94,34 \\
\end{tabular} & \begin{tabular}{|l|l|}
2,80 & 0,74 \\
\end{tabular} & 94,34 & $39,1 \pm 0,4$ & 3,52 \\
\hline 2 & \begin{tabular}{|l|l|}
2,10 & 94,56 \\
\end{tabular} & \begin{tabular}{|l|l|}
2,67 & 0,67 \\
\end{tabular} & 94,56 & $43,3 \pm 0,3$ & 3,56 \\
\hline 3 & \begin{tabular}{|l|l|}
2,06 & 95,16 \\
\end{tabular} & \begin{tabular}{|l|l|}
2,12 & 0,66 \\
\end{tabular} & 95,16 & $44,7 \pm 0,3$ & 3,58 \\
\hline 4 & \begin{tabular}{|l|l|}
2,03 & 95,87 \\
\end{tabular} & \begin{tabular}{|l|l|}
1,59 & 0,51 \\
\end{tabular} & 95,87 & $44,8 \pm 0,3$ & 3,61 \\
\hline 5 & \begin{tabular}{|l|l|}
3,62 & 94,46 \\
\end{tabular} & \begin{tabular}{|l|l|}
1,37 & 0,55 \\
\end{tabular} & 94,46 & $51,1 \pm 0,2$ & 3,60 \\
\hline
\end{tabular}

Было установлено, что используемые водные растворы метасиликата натрия и фосфата натрия в качестве связующих оказывают влияние на процесс гранулообразования. Значительное увеличе- 
ние статической прочности достигается при использовании фосфата натрия, разница между максимальными значениям достигнутыми при этих связующих составляет $27 \mathrm{H} /$ гранула. Однако применение таких концентрированных растворов может привести к увеличению себестоимости готового продукта [21].

Влияние связующих веществ объясняется образованием новых фаз в объеме гранул биогумуса за счет протекания химических реакций между компонентами удобрения. При проведении термодинамического анализа с использованием программного обеспечения «HSC 6.0 » была оценена возможность протекания данных реакций, представленных в табл. 3.

При этом были рассчитаны значения теплового эффекта $\left(\Delta H_{\text {х.p. }}^{\circ}\right)$, изменение энтропии $\left(\Delta S_{\text {х.р. }}^{\circ}\right)$, изменения энергии Гиббса $\left(\Delta G_{\mathrm{x} . \mathrm{o} .}\right)$ и константы равновесия $\left(K_{\mathrm{p}}\right)$.

Установлено, что протекание данных реакций возможно в условиях проведения процесса сушки и гранулирования биогумуса. В ходе расчетов определили вероятность образования труднорастворимых солей: $\mathrm{MgSiO}_{3}, \mathrm{CaSiO}_{3}, \mathrm{FeSiO}_{3}, \mathrm{AlPO}_{4}$, $\mathrm{Mg}_{3}\left(\mathrm{PO}_{4}\right)_{2}, \mathrm{Ca}_{3}\left(\mathrm{PO}_{4}\right)_{2}, \mathrm{Fe}_{3}\left(\mathrm{PO}_{4}\right)_{2}$, которые способствуют дополнительной цементации гранул, увеличивая их прочность. Данные соединения не растворимы в воде и в условиях термической обработки материала, кристаллизуются в виде микрокристаллов [22]. Эти кристаллы выступают в роли центров кристаллообразования и способствуют формированию солевых мостиков. Благодаря этому происходит усиленное сцепление мелких частиц удобрения и образование упрочненной структуры гранулы.

В процессе гранулирования биогумуса образуется часть мелкой (менее 2,0 мм) и крупной (более 5,5 мм) фракции, которая не соответствует требуемым характеристикам продукта. Представляется возможность использования некондиционной фракции в качестве ретура, которая после измельчения смешивается с формуемой тукосмесью. Важнейшим параметром гранулирования биогу-

Таблица 4. Терлодинамические константы равновесия реакиий, протекающих между связующими и примесями, присутствующими в органоминеральном удобрении

Table 4. Thermodynamic equilibrium constants of reactions between binders and impurities in organomineral fertilizers

\begin{tabular}{|c|c|c|c|c|c|c|c|c|c|}
\hline$T,{ }^{\circ} \mathrm{C}$ & $\Delta H_{\mathrm{x} . \mathrm{p}}^{\circ}, \mathrm{\kappa Д}$ & $\Delta S_{\text {x.p }}^{\circ}$ кДж & $\Delta G_{\mathrm{x} . \mathrm{p},}^{\circ} \mathrm{\kappa}$ Һж & $K_{\mathrm{p}}$ & $T,{ }^{\circ} \mathrm{C}$ & $\Delta H_{\text {x.p. }}^{\circ}, \kappa Д ж$ & $\Delta S_{\text {x.p }}^{\circ}$ кДж & $\Delta G_{\mathrm{x} . \mathrm{p},}^{\circ}$ кДж & $K_{\mathrm{p}}$ \\
\hline \multicolumn{5}{|c|}{$\mathrm{Na}_{2} \mathrm{SiO}_{3} \cdot 5 \mathrm{H}_{2} \mathrm{O}+\mathrm{CaCl}_{2}=\mathrm{CaSiO}_{3} \downarrow+2 \mathrm{NaCl}+5 \mathrm{H}_{2} \mathrm{O}$} & \multicolumn{5}{|c|}{$2 \mathrm{Na}_{3} \mathrm{PO}_{4} \cdot 12 \mathrm{H}_{2} \mathrm{O}+3 \mathrm{CaCl}_{2}=6 \mathrm{NaCl}+\mathrm{Ca}_{3}\left(\mathrm{PO}_{4}\right)_{2} \downarrow+24 \mathrm{H}_{2} \mathrm{O}$} \\
\hline 25 & $-57,540$ & 86,821 & $-83,425$ & $4,140 \mathrm{E}+014$ & 25 & $-100,679$ & 700,343 & $-309,487$ & $1,680 \mathrm{E}+054$ \\
\hline 50 & $-53,889$ & 98,671 & $-85,281$ & $1,006 \mathrm{E}+014$ & 50 & $-80,882$ & 764,103 & $-327,801$ & $9,793 \mathrm{E}+052$ \\
\hline 70 & $-50,147$ & 110,077 & $-87,369$ & $3,142 \mathrm{E}+013$ & 70 & $-64,891$ & 812,115 & $-343,568$ & $2,007 \mathrm{E}+052$ \\
\hline 90 & $-46,321$ & 121,067 & $-89,681$ & $1,204 \mathrm{E}+013$ & 90 & $-48,738$ & 857,864 & $-360,271$ & $6,681 \mathrm{E}+051$ \\
\hline 110 & $-42,409$ & 131,694 & $-92,209$ & $5,472 \mathrm{E}+012$ & 110 & $-32,357$ & 901,771 & $-377,870$ & $3,305 \mathrm{E}+051$ \\
\hline \multicolumn{5}{|c|}{$\mathrm{Na}_{2} \mathrm{SiO}_{3} \cdot 5 \mathrm{H}_{2} \mathrm{O}+\mathrm{FeCl}_{2}=\mathrm{FeSiO}_{3} \downarrow+2 \mathrm{NaCl}+5 \mathrm{H}_{2} \mathrm{O}$} & \multicolumn{5}{|c|}{$\mathrm{Na}_{3} \mathrm{PO}_{4} \cdot 12 \mathrm{H}_{2} \mathrm{O}+\mathrm{FeCl}_{3}=\mathrm{FePO}_{4} \downarrow+3 \mathrm{NaCl}+12 \mathrm{H}_{2} \mathrm{O}$} \\
\hline 25 & $-70,975$ & 91,162 & $-98,155$ & $1,577 \mathrm{E}+017$ & 25 & $-84,022$ & 340,646 & $-185,586$ & $3,285 \mathrm{E}+032$ \\
\hline 50 & $-67,290$ & 103,122 & $-100,099$ & $2,728 \mathrm{E}+016$ & 50 & $-76,737$ & 364,111 & $-194,400$ & $2,665 \mathrm{E}+031$ \\
\hline 70 & $-63,526$ & 114,595 & $-102,276$ & $6,312 \mathrm{E}+015$ & 70 & $-70,911$ & 381,605 & $-201,858$ & $5,366 \mathrm{E}+030$ \\
\hline 90 & $-59,688$ & 125,621 & $-104,679$ & $1,855 \mathrm{E}+015$ & 90 & $-65,047$ & 398,211 & $-209,658$ & $1,443 \mathrm{E}+030$ \\
\hline 110 & $-54,778$ & 138,871 & $-107,987$ & $5,284 \mathrm{E}+014$ & 110 & $-59,102$ & 414,147 & $-217,782$ & $4,928 \mathrm{E}+029$ \\
\hline \multicolumn{5}{|c|}{$\mathrm{Na}_{2} \mathrm{SiO}_{3} \cdot 5 \mathrm{H}_{2} \mathrm{O}+\mathrm{MgCl}_{2}=2 \mathrm{NaCl}+\mathrm{MgSiO}_{3} \downarrow+5 \mathrm{H}_{2} \mathrm{O}$} & \multicolumn{5}{|c|}{$2 \mathrm{Na}_{3} \mathrm{PO}_{4} \cdot 12 \mathrm{H}_{2} \mathrm{O}+3 \mathrm{MgCl}_{2}=6 \mathrm{NaCl}+\mathrm{Mg}_{3}\left(\mathrm{PO}_{4}\right)_{2} \downarrow+24 \mathrm{H}_{2} \mathrm{O}$} \\
\hline 25 & $-121,198$ & 94,809 & $-149,939$ & $6,880 \mathrm{E}+025$ & 25 & $-213,149$ & 709,789 & $-424,772$ & $2,658 \mathrm{E}+074$ \\
\hline 50 & $-117,220$ & 107,516 & $-151,964$ & $3,679 \mathrm{E}+024$ & 50 & $-193,522$ & 772,995 & $-443,315$ & $4,617 \mathrm{E}+071$ \\
\hline 70 & $-113,217$ & 119,535 & $-154,235$ & $3,018 \mathrm{E}+023$ & 70 & $-177,570$ & 820,887 & $-459,258$ & $8,213 \mathrm{E}+069$ \\
\hline 90 & $-109,183$ & 130,960 & $-156,741$ & $3,525 \mathrm{E}+022$ & 90 & $-161,386$ & 866,724 & $-476,137$ & $3,105 \mathrm{E}+068$ \\
\hline 110 & $-105,104$ & 141,893 & $-159,470$ & $5,525 \mathrm{E}+021$ & 110 & $-144,915$ & 910,871 & $-493,915$ & $2,192 \mathrm{E}+067$ \\
\hline \multicolumn{5}{|c|}{$\mathrm{Na}_{2} \mathrm{SiO}_{3} \cdot 5 \mathrm{H}_{2} \mathrm{O}+\mathrm{MgSO}_{4}=\mathrm{Na}_{2} \mathrm{SO}_{4}+\mathrm{MgSiO}_{3} \downarrow+5 \mathrm{H}_{2} \mathrm{O}$} & \multicolumn{5}{|c|}{$2 \mathrm{Na}_{3} \mathrm{PO}_{4} \cdot 12 \mathrm{H}_{2} \mathrm{O}+3 \mathrm{MgSO}_{4}=3 \mathrm{Na}_{2} \mathrm{SO}_{4}+\mathrm{Mg}_{3}\left(\mathrm{PO}_{4}\right)_{2} \downarrow+24 \mathrm{H}_{2} \mathrm{O}$} \\
\hline 25 & $-70,349$ & 94,850 & $-98,628$ & $1,908 \mathrm{E}+017$ & 25 & $-57,630$ & 719,796 & $-272,237$ & $4,997 \mathrm{E}+047$ \\
\hline 50 & $-65,325$ & 111,028 & $-101,204$ & $2,292 \mathrm{E}+016$ & 50 & $-37,839$ & 783,531 & $-291,037$ & $1,116 \mathrm{E}+047$ \\
\hline 70 & $-61,270$ & 123,203 & $-103,547$ & $5,800 \mathrm{E}+015$ & 70 & $-21,731$ & 831,891 & $-307,194$ & $5,825 \mathrm{E}+046$ \\
\hline 90 & $-57,172$ & 134,811 & $-106,128$ & $1,847 \mathrm{E}+015$ & 90 & $-5,353$ & 878,275 & $-324,299$ & $4,470 \mathrm{E}+046$ \\
\hline 110 & $-53,013$ & 145,958 & $-108,937$ & $7,121 \mathrm{E}+014$ & 110 & 11,358 & 923,066 & $-342,315$ & $4,693 \mathrm{E}+046$ \\
\hline \multicolumn{5}{|c|}{$\mathrm{Na}_{2} \mathrm{SiO}_{3} \cdot 5 \mathrm{H}_{2} \mathrm{O}+\mathrm{FeSO}_{4}=\mathrm{Na}_{2} \mathrm{SO}_{4}+\mathrm{FeSiO}_{3} \downarrow+5 \mathrm{H}_{2} \mathrm{O}$} & \multicolumn{5}{|c|}{$2 \mathrm{Na}_{3} \mathrm{PO}_{4} \cdot 12 \mathrm{H}_{2} \mathrm{O}+3 \mathrm{FeSO}_{4}=3 \mathrm{Na}_{2} \mathrm{SO}_{4}+\mathrm{Fe}_{3}\left(\mathrm{PO}_{4}\right)_{2} \downarrow+24 \mathrm{H}_{2} \mathrm{O}$} \\
\hline 25 & $-49,087$ & 93,578 & $-76,988$ & $3,083 \mathrm{E}+013$ & 25 & $-80,853$ & $-392,964$ & 36,309 & $4,348 \mathrm{E}-007$ \\
\hline 50 & $-44,406$ & 108,654 & $-79,517$ & $7,152 \mathrm{E}+012$ & 50 & $-66,900$ & $-348,025$ & 45,564 & $4,308 \mathrm{E}-008$ \\
\hline 70 & $-40,584$ & 120,128 & $-81,806$ & $2,842 \mathrm{E}+012$ & 70 & $-55,698$ & $-314,391$ & 52,185 & $1,137 \mathrm{E}-008$ \\
\hline 90 & $-36,688$ & 131,163 & $-84,319$ & $1,347 \mathrm{E}+012$ & 90 & $-44,402$ & $-282,398$ & 58,151 & $4,316 \mathrm{E}-009$ \\
\hline 110 & $-32,704$ & 141,841 & $-87,050$ & $7,387 \mathrm{E}+011$ & 110 & $-32,927$ & $-251,641$ & 63,489 & $2,207 \mathrm{E}-009$ \\
\hline \multicolumn{5}{|c|}{$\mathrm{Na}_{2} \mathrm{SiO}_{3} \cdot 5 \mathrm{H}_{2} \mathrm{O}+\mathrm{CaSO}_{4}=\mathrm{Na}_{2} \mathrm{SO}_{4}+\mathrm{CaSiO}_{3} \downarrow+5 \mathrm{H}_{2} \mathrm{O}$} & \multicolumn{5}{|c|}{$2 \mathrm{Na}_{3} \mathrm{PO}_{4} \cdot 12 \mathrm{H}_{2} \mathrm{O}+3 \mathrm{CaSO}_{4}=3 \mathrm{Na}_{2} \mathrm{SO}_{4}+\mathrm{Ca}_{3}\left(\mathrm{PO}_{4}\right)_{2} \downarrow+24 \mathrm{H}_{2} \mathrm{O}$} \\
\hline 25 & 19,033 & 93,813 & $-8,938$ & $3,681 \mathrm{E}+001$ & 25 & 129,037 & 721,318 & $-86,024$ & $-86,024$ \\
\hline 50 & 23,605 & 108,538 & $-11,469$ & $7,145 \mathrm{E}+001$ & 50 & 148,818 & 785,027 & $-104,863$ & $8,948 \mathrm{E}+016$ \\
\hline 70 & 27,346 & 119,767 & $-13,753$ & $1,241 \mathrm{E}+002$ & 70 & 164,740 & 832,831 & $-121,046$ & $2,675 \mathrm{E}+018$ \\
\hline 90 & 31,163 & 130,578 & $-16,257$ & $2,180 \mathrm{E}+002$ & 90 & 180,805 & 878,331 & $-138,161$ & $7,489 \mathrm{E}+019$ \\
\hline 110 & 35,069 & 141,049 & $-18,973$ & $3,862 \mathrm{E}+002$ & 110 & 197,102 & 922,011 & $-156,167$ & $1,959 \mathrm{E}+021$ \\
\hline
\end{tabular}


муса является количество используемого ретура, а также его влияние на характеристики гранул биогумуса [23].

Были проведены опыты по изучению влияния введения предварительно измельченной до менее 1,0 мм нетоварной фракции в формуемую смесь. Количество содержания ретура в исходной смеси составляло 1, 3 и 5 \% к общей массе порошкообразного удобрения (табл. 5).

Таблица 5. Характеристики гранул биогумуса в зависимости от величины вводилого ретура

Table 5. Characteristics of biohumus granules depending on the size of the introduced retour

\begin{tabular}{|c|c|c|c|c|c|c|c|}
\hline \multirow{3}{*}{ 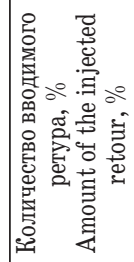 } & \multicolumn{4}{|c|}{$\begin{array}{c}\text { Гранулометрический } \\
\text { состав, \% } \\
\text { Granulometric } \\
\text { composition, \% }\end{array}$} & \multirow{3}{*}{ 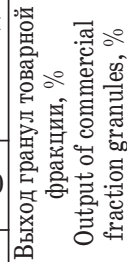 } & \multirow{3}{*}{ 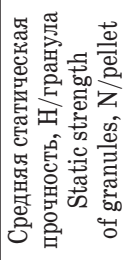 } & \multirow{3}{*}{ 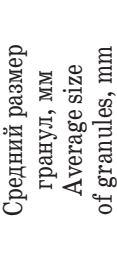 } \\
\hline & $+5,5$ & $\begin{array}{l}-5,5 \\
+2,0\end{array}$ & $\begin{array}{l}-2,0 \\
+1,0\end{array}$ & $-1,0$ & & & \\
\hline & \multicolumn{4}{|c|}{$\mathrm{MM} / \mathrm{mm}$} & & & \\
\hline 1 & 3,09 & 94,04 & 2,63 & 0,24 & 94,04 & $28,4 \pm 0,2$ & 3,75 \\
\hline 3 & 1,99 & 94,09 & 2,93 & 0,99 & 94,09 & $27,2 \pm 0,2$ & 3,70 \\
\hline 5 & 2,12 & 93,96 & 2,72 & 1,20 & 93,96 & $26,3 \pm 0,3$ & 3,70 \\
\hline
\end{tabular}

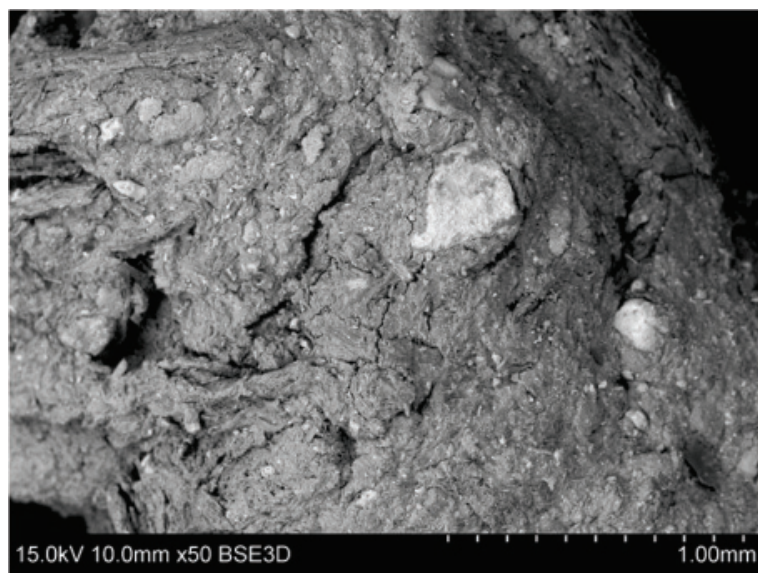

Pис. 4. Микрофотография поверхности гранулы биогулуса (увеличение 250$)$.

Fig. 4. Microphotography of biohumus granule surface (magnifica tion $\times 50$ )

С увеличением количества ретура от 1 до $5 \%$ незначительно уменьшается выход товарной фракции, а прочность гранул снижается на 2 Н/гранулу. Такое поведение можно объяснить с точки зрения взаимодействия связующего раствора с тукосмесью. Так как при добавлении ретура в исходную смесь изменяется гранулометрический состав и способность смачивания сухих частиц ретура различны, содержание вводимого связующего реагента становится недостаточным. Увеличение содержания ретура в исходной смеси требует большего количества вводимого в тукосмесь связующего раствора, что приведет к дополнительным затратам. В данном случае для более рационального метода гранулирования окатыванием следует вводить ретур с содержанием 3 \% к массе тукосмеси, благо- даря чему выход гранул товарной фракции и прочность гранулята составит 94,09% и 27 Н/гранула, соответственно.

Изображение поверхности гранулы биогумуса, полученной методом окатывания, представлено на рис. 4.

Видно, что на поверхности гранулы частицы склеены между собой и образуют уплотненную упаковку. Наблюдаются трещины размером 0,01-0,05 мм, образовавшиеся в процессе сушки, а также волокнистая структура биоматериала, который является компонентом биогумуса. На поверхности присутствуют крупные вкрапления светлого цвета размером 0,2-0,4 мм.

В результате проведенного элементного анализа установлено, что входящие в состав биогумуса различные по размеру светлые частицы могут быть $\mathrm{Al}_{2} \mathrm{O}_{3}$ и $\mathrm{SiO}_{2}$.

\section{Заключение}

Благодаря исследованиям процесса гранулирования биогумуса были определены оптимальные технологические параметры проведения данного процесса. Гранулирование биогумуса методом окатывания с предварительным формованием через ячейки с диаметром 4,0 мм следует проводить при температуре $25^{\circ} \mathrm{C}$ в течение $180 \mathrm{c}$.

Оптимальное количество вводимого ретура в тукосмесь ОМУ составляет $3 \%$, а повешение его содержания требует дополнительной подачи раствора связующего.

В качестве связующих веществ использовались водные растворы метасиликата и фосфата натрия с разными концентрациями. На основании проведенного термодинамического анализа установлено, что вероятность взаимодействия фосфата натрия с компонентами биогумуса выше, а в результате протекающих химических реакций образуются труднорастворимые соединения. Образованные $\mathrm{Mg}_{3}\left(\mathrm{PO}_{4}\right)_{2}, \mathrm{Ca}_{3}\left(\mathrm{PO}_{4}\right)_{2}, \mathrm{Fe}_{3}\left(\mathrm{PO}_{4}\right)_{2}$, способствуют дополнительному упрочнению гранул за счет кристаллизации данных солей в виде микрокристаллов. Использование водного раствора фосфата натрия приводит к улучшению товарных характеристик готового продукта: выход гранул фракции $+5,5-2,0$ мм и статическая прочность составляют $94,46 \%$ и $51 \mathrm{H} /$ гранула, соответственно.

С помощью термодинамического анализа установлена вероятность образования труднорастворимых соединений в результате взаимодействия водных растворов связующих с компонентами органоминерального удобрения, таких как $\mathrm{MgSiO}_{3}, \mathrm{CaSiO}_{3}$, $\mathrm{FeSiO}_{3}, \mathrm{AlPO}_{4}, \mathrm{Mg}_{3}\left(\mathrm{PO}_{4}\right)_{2}, \mathrm{Ca}_{3}\left(\mathrm{PO}_{4}\right)_{2}, \mathrm{Fe}_{3}\left(\mathrm{PO}_{4}\right)_{2}$. Образовавшиеся соединения не растворимы, в процессе сушки кристаллизуются в виде микрокристаллов, выступающих в роли центров кристаллообразования, что способствует формированию прочных солевых мостиков. Это приводит к усилению сцепления между частицами удобрения и образованию упрочненной и уплотненной структуры в гранулах. 


\section{СПИСОК ЛИТЕРАТУРЫ}

1. Eartworm biohumus conditioning for pellet production / 0. Vronskis, A. Kakitis, E. Laukmanis, I. Nulle // Engineering for rural development. - 2016. - V. 25. - P. 997-1002.

2. Characterisation of organomineral fertilisers derived from nutrient-enriched biosolids granules / L.A. Diogenes, R. Sakrabani, F.T. Sean, M.S. Le, R.J. Godwin // Applied and Environmental Soil Science. - 2013. - V. 2013. - P. 1-11.

3. Tumuluru J.S. Effect of process variables on density and durability of pellets made from high moisture corn stover // Biosystems Engineering. - 2013. - V. 119. - P. 44-57.

4. Состав для производства органоминерального удобрения: пат. Рос. Федерация, № 2566048, заявл. 26.09.2014; опубл. 20.10.2015. Бюл. № 33. -5 с.

5. Способ получения комплексного органоминерального удобрения из отходов солодки голой и технологическая линия для его осуществления: пат. Рос. Федерация, № 2221761, заявл. 12.08.2002; опубл. 20.01.2004. Бюл. № 4. - 11 с.

6. Состав органоминеральных удобрений и способ их получения: пат. Рос. Федерация, № 2574688, заявл. 16.09.2014; опубл. 10.02.2016. Бюл. № 4. -6 с.

7. Органоминеральное гранулированное удобрение и способ его получения: пат. Рос. Федерация, № 2491263, заявл. 02.04.2012; опубл. 27.08.2013. Бюл. № 24. - 6 с.

8. Многокомпонентное органоминеральное удобрение: пат. Рос. Федерация, № 2566684, заявл. 20.11.2014; опубл. 27.10.2015. Бюл. № 30. - 7 с.

9. Аллахвердиев С.Р., Ерошенко В.И. Современные технологии в органическом земледелии // Международный журнал прикладных и фундаментальных исследований. - 2017. - № 1. C. 76-79.

10. Гранулированное удобрение на основе торфа: пат. Рос. Федерация, № 2469994 заявл. 19.07.2011; опубл. 20.12.2012. Бюл. № $35 .-21 \mathrm{c}$.

11. Способ изготовления гранулированного органоминерального удобрения: пат. Рос. Федерация, № 2272800 заявл. 29.07.2004; опубл. 27.03.2006. Бюл. № 9. - 5 с.

12. Способ получения комплексного органоминерального удобрения: пат. Рос. Федерация, № 2219147 заявл. 14.03.2002; опубл. 20.12.2003. Бюл. № 36. - 9 с.

13. Сковородников П.В., Черепанова М.В. Способы гранулирования органоминеральных удобрений // Вестник ПНИПУ. Химическая технология и биотехнология. - 2017. - № 3. C. $117-127$.

\section{Информация об авторах}

Сковородников П.В., магистр кафедры химических технологий Химико-технологического факультета Пермского национального исследовательского политехнического университета.

Черепанова M.В., кандидат технических наук, доцент кафедры химических технологий Химико-технологического факультета Пермского национального исследовательского политехнического университета.
14. Першин В.Ф. Переработка сыпучих материалов в машинах барабанного типа. - М.: Машиностроение, 2009 - 216 с.

15. Макаренко Д.А., Назаров В.И. Особенности гранулирования комплексных и органоминеральных удобрений в скоростных и тарельчатых грануляторах окатывания // Вестник МГОУ. Естественные науки. - 2015. - № 4. - С. 81-88.

16. Investigation of granulation process parameters influence on granulated fertilizer compost properties / A. Pocius, E. Jotautiene, R. Mieldazys, A. Jasinskas, V. Kucinskas // Engineering for Rural Development: Proceedings of 13 International Conference. Jelgava, Latvia, 2014. - P. 407-412.

17. Zafari A., Kianmehr M.H. Factors affecting mechanical properties of biomass pellet from compost // Environmental Technology. 2013. - V. 24. - P. 1-9.

18. Сковородников П.В., Черепанова М.В. Изучение влияния содержания водного раствора связующего тринатрийфосфата на характеристики гранулированного биогумуса полученного методом окатывания // Химия. Экология. Урбанистика: Материалы Всероссийской научно-практической конференции (с международным участием). - Пермь: ПНИПУ, 2018. C. $770-774$.

19. Effect of moisture content particle size and pine addition on quality parameters of barley straw pellets / C. Serrano, E. Monedero, M. Laupuerta, H. Portero // Fuel Processing Technology. 2011. - V. 92. - P. 699-706.

20. Optimization compressive strength biomass pellet from compost using Taguchi method / G. Absalan, M.H. Kianmehr, A. Arabhosseini, S. Kouravand // Agricultural Engineering International. 2015. - V. 17. - P. 166-172.

21. Кувшинников И.М. Минеральные удобрения и соли. Свойства и способы улучшения. - М.: Химия, 1987 - 256 с.

22. Investigation of physical-mechanical properties of experimental organic granular fertilizers / A. Pocius, E. Jotautiene, J. Pekarskas, M. Palsauskas // Engineering for rural development. -

23. Макаренко Д.А., Назаров В.И. Исследование процесса гранулирования комплексных удобрений с учетом физико-химических и реологических свойств компонентов // Вестник МГОУ. Естественные науки. - 2012. - № 2. - С. 49-55.

Поступила 27.08.2019 2. 2016. - V. 25. - P. 1116-1120.

ческого факультета ПІермского национального исследовательского политехнического университета. 
UDC 661.152 .4

\title{
PECULIARITIES OF ORGANOMINERAL FERTILIZER GRANULATION BY THE PELLETIZING METHOD
}

\section{Pavel V. Skovorodnikov',}

pavel.skovorodnikov@yandex.ru

\author{
Maria V. Cherepanova', \\ syromyatnikova.maria@yandex.ru \\ 1 Perm National Research Polytechnic University, \\ 29, Komsomolsky avenue, Perm, 614990, Russia.
}

\begin{abstract}
The relevance. The use of organomineral fertilizers makes it possible to substantially increase the fertility of soil and restore its agrochemical and physical properties. Research allows you to solve some problems associated with fertilizer application in untreated form, and the resulting product in the form of spherical granules measuring of 2,0 to 5,5 $\mathrm{mm}$ will have a high static strength and can be easily used in agriculture in conditions of continuous application of fertilizers to the soil with the use of agrotechnics.

The main aim of the research is to study the organomineral fertilizer granulation by pelletizing with preliminary subforming and determination of optimal technological parameters; to investigate the probability of formation of sparingly soluble compounds in interaction of solutions of binders with components that are part of organomineral fertilizers.

Objects: organomineral fertilizer biohumus with a moisture content of 55-60 \%

Methods: electronic scanning microscopy (Hitachi «S-3400N»), which allows determining the elemental composition of biohumus and evaluating the surface of fertilizer particles and the finished granular product; $X$-ray spectral analysis used to establish the elemental composition; the thermodynamic analysis necessary for estimation of probability of proceeding the reactions and possibility of formation of difficult-to-constructible compounds in the granular fertilizer, promoting increase in strength of granules; sieve analysis, used to determine the particle size distribution, and a method for determining the static strength of granules on an IPG-1M instrument.

Results. Using the electron-scanning microscope, the surface of organic-mineral fertilizer particles - biohumus - was studied and described. The elemental composition of organomineral fertilizer is determined: C, N, P, K, O, Fe, Al, Ca, Mg, Na, Cl, S, Ti. Optimum parameters of the technology of organomineral fertilizer granulation with preliminary molding through cells with a diameter of 4,0 $\mathrm{mm}$ were determined: duration of $180 \mathrm{~s}$, rolling temperature of $25{ }^{\circ} \mathrm{C}$, drying at $110{ }^{\circ} \mathrm{C}$ for $60 \mathrm{~min}$, and retention value of $3 \%$. The type and content of the binder solution in the fertilizer mixture is determined, which makes it possible to obtain the product with the best commercial characteristics (static strength, output of product fraction of 2,0 to 5,5 $\mathrm{mm}$ ). Based on the thermodynamic analysis, the possibility of chemical reactions between the binder solution and biohumus components was established, resulting in formation of sparingly soluble compounds $\left(\mathrm{Mg}_{3}\left(\mathrm{PO}_{4}\right)_{2}, \mathrm{Ca}_{3}\left(\mathrm{PO}_{4}\right)_{2}, \mathrm{Fe}_{3}\left(\mathrm{PO}_{4}\right)_{2}\right)$, which increase the strength of the granules. The sodium phosphate aqueous solution used as a binder allows obtaining pellets having a high static strength (51,1 N/pellet). With the help of electron-scanning microscopy, the structure of the obtained granular fertilizer was studied.
\end{abstract}

Key words:

Granulation, organomineral fertilizer, biohumus, pelletizing, binder solution, natrium metasilicate, sodium phosphate.

\section{REFERENCES}

1. Vronskis 0., Kakitis A., Laukmanis E., Nulle I. Eartworm biohumus conditioning for pellet production. Engineering for rural development, 2016, vol. 25, pp. 997-1002.

2. Diogenes L.A., Sakrabani R., Sean F.T., Minh S., Richard J.G. Characterisation of organomineral fertilisers derived from nutrient-enriched biosolids granules. Applied and Environmental Soil Science, 2013, vol. 2013, pp. 1-11.

3. Tumuluru J.S. Effect of process variables on density and durability of pellets made from high moisture corn stover. Biosystems Engineering, 2013, vol. 119, pp. 44-57.

4. Pegushin S.N., Voxmyanin V.G. Sostav dlya proizvodstva organo mineralnogo udobreniya [Composition for production organic-mineral fertilizer]. Patent RF, no. 2566048, 2015.

5. Rogachev A.F., Saldaev A.M., Mazaeva T.I. Sposob polucheniya kompleksnogo organomineralnogo udobreniya iz otkhodov solodki goloy i tekhnologicheskaya liniya dlya ego osushchestuleniya [The method of obtaining complex organic-mineral fertilizers from waste licorice and technological line for its implementation]. $\mathrm{Pa}$ tent RF, no. 2221761, 2004.

6. Bobritskii G.A., Kladov A.A., Frolov G.V. Sostav organomineral nykh udobreniy $i$ sposob ikh polucheniya [The composition of the organic-mineral fertilizers and the method their production]. Patent RF, no. 2574688, 2016.

7. Stifeev A.I., Zolotukhin L.A. Organomineralnoe granulirovannoe udobrenie i sposob ego polucheniya [Organic-mineral granular fer- tilizer and the method of its production]. Patent RF, no. $2491263,2013$.

8. Vazykhov I.T., Kireicheva L.V., Pukhovskaya T.Yu., Pavlov V.Yu. Mnogokomponentnoe organomineralnoe udobrenie [Multicomponent organic-mineral fertilizer]. Patent RF, no. $2566684,2015$.

9. Allahverdiev S.R., Eroshenko V.I. Modern technology in organic farming. International journal of applied and fundamental research, 2017, no. 1, pp. 76-79. In Rus.

10. Kormilitsyn V.I., Lobko V.P. Granulirovannoe udobrenie na osnove torfa [Peat-based granular fertiliser]. Patent RF, no. 2469994, 2012.

11. Zhivopistsev V.A., Malanchuk V.Ya., Mikhaylov G.V. Sposob izgotovleniya granulirovannogo organomineralnogo udobreniya [Method of manufacturing granulated organomineral fertilizer]. Patent RF, no. 2272800, 2006.

12. Kovalev K.E., Papayanaki V.V. Sposob polucheniya kompleksnogo organomineralnogo udobreniya [The method of obtaining complex organic-mineral fertilizers]. Patent RF, no. 2219147, 2003.

13. Skovorodnikov P.V., Cherepanova M.V. The methods of granulation organomineral fertilizers. Bulletin of the Perm national research polytechnic university, 2017, no. 3, pp. 39-49. In Rus.

14. Pershin V.F. Pererabotka sypuchikh materialov v mashinakh barabannogo tipa [Bulk material processing in drum-type machines]. Moscow, Mashinostroenie Publ., 2009. 216 p.

15. Makarenko D.A., Nazarov V.I. Features of the granulation process of complex and organic fertilizers in high-speed granulation 
ad dish nodulizers. Bulletin of the Moscow region state university, 2015, no. 4, pp. 81-88. In Rus.

16. Pocius A., Jotautiene E., Mieldazys R., Jasinskas A., Kucinskas V. Investigation of granulation process parameters influence on granulated fertilizer compost properties. Engineering for Rural Development. Proceedings of 13 International Conference. Jelgava, Latvia, 2014. pp. 407-412.

17. Zafari A., Kianmehr M.H. Factors affecting mechanical properties of biomass pellet from compost. Environmental Technology, 2013, vol. 24, pp. 1-9.

18. Skovorodnikov P.V., Cherepanova M.V. Izuchenie vliyaniya soderzhaniya vodnogo rastvora svyazuyushchego trinatriyfosfata na kharakteristiki granulirovannogo biogumusa, poluchennogo metodom okatyvaniya [Studying the influence of the content of the trisodium phosphate binding water solution on characteristics of the granulated biogumus obtained by the pelletizing method]. Khimiya. Ekologiya. Urbanistika. Materialy Vserossiyskoy nauchno-prakticheskoy konferentsii (s mezhdunarodnym uchastiem ) [Chemistry. Ecology. Urbanism. Materials of the AllRussian scientific-practical conference (with international participation)]. Perm, PNRPU Publ., 2018. pp. 770-774.
19. Serrano C., Monedero E., Laupuerta M., Portero H. Effect of moisture content particle size and pine addition on quality parameters of barley straw pellets. Fuel Processing Technology, 2011, vol. 92, pp. 699-706.

20. Absalan G., Kianmehr M.H., Arabhosseini A., Kouravand S. Optimization compressive strength biomass pellet from compost using Taguchi method. Agricultural Engineering International, 2015, vol. 17, pp. 166-172.

21. Kuvshinnikov I.M. Mineralnye udobreniya i soli. Svoystva i sposoby uluchsheniya [Mineral fertilizers and salts. Properties and methods of improvement]. Moscow, Khimiya Publ., 1987. 256 p. 22. Pocius A., Jotautiene E., Pekarskas J., Palsauskas M. Investigation of physical-mechanical properties of experimental organic granular fertilizers. Engineering for rural development, 2016, vol. 25, pp. 1116-1120.

23. Makarenko D.A., Nazarov V.I. Study of process of granulation of complex fertilizers taking into account physical-chemical and rheological properties of components. Bulletin of the Moscow region state university, 2012, no. 2, pp. 49-54. In Rus.

Received: 27 August 2019.

\section{Information about the authors}

Pavel V. Skovorodnikov, master, Perm National Research Polytechnic University.

Maria V. Cherepanova, Cand. Sc., associate professor, Perm National Research Polytechnic University. 